\title{
Osteoporosis is associated with a lower prevalence of body mass index-defined obesity in rheumatoid arthritis
}

\author{
Catalina Raluca Nuta ${ }^{1,2}$, Claudiu C. Popescu ${ }^{2,3,{ }^{*}}$, Denisa Predeteanu ${ }^{2,4}$, \\ Ruxandra lonescu ${ }^{2,4}$ \\ 1"Ana Aslan" National Institute of Gerontology and Geriatrics, Bucharest, Romania \\ 2"Carol Davila" University of Medicine and Pharmacy, Bucharest, Romania \\ 3"Dr. Ion Stoia" Clinical Centre for Rheumatic Diseases, Bucharest, Romania \\ 4"Sf. Maria" Clinical Hospital, Bucharest, Romania
}

\begin{abstract}
Objective. The objective of this initial phase of the study is to retrospectively screen rheumatoid arthritis (RA) phenotype characteristics associated with osteoporosis.

Methods. The study included all RA patients who randomly came to the university rheumatology department between January and July 2018. Demographic data, anthropometric data, RA-specific variables, osteoporosis data and comorbidities were collected retrospectively and cross-sectionally from the first (and most frequently the only) observation sheet of each patient within the study timeframe. Correlations and comparison were analyzed using appropriate non-parametric tests, all of the reported being significant $(p<0.05)$.

Results. The sample included 149 RA patients (60.8 years mean age; $81.2 \%$ women), 40 (26.8\%) of which had osteoporosis and 31 (20.8\%) were obese. Compared to RA patients without osteoporosis, RA patients with osteoporosis were significantly older (56.0 respectively 71.0 years) and had: lower body mass index (BMl; $23.8 \mathrm{~kg} / \mathrm{m}^{2}$ respectively $29.6 \mathrm{~kg} / \mathrm{m}^{2}$ ), longer disease duration (11.0 respectively 17.0 years), higher prevalence of rural dwelling (prevalence ratio $-P R=2.46)$, smoking $(P R=3.71)$, inflammation $(P R=1.35)$, anti-citrullinated protein antibody positivity $(P R=1.51)$, glucocorticoids $(P R=1.85)$ and carotid artery disease $(P R=3.01)$, but a lower prevalence of obesity (PR=3.43). Lumbar bone mineral density was significantly correlated with BMI (rho=0.294) and with rheumatoid factor titers (rho=0.311), controlling for age, gender and disease duration.

Conclusions. BMI-defined obesity seems to be associated with a lower prevalence of osteoporosis among RA patients, while disease severity (treatment with glucocorticoids, inflammation and ACPA positivity) is associated with a higher prevalence of osteoporosis. Gain of adipose tissue and loss of bone tissue seem to be antagonistic and parallel body composition alterations in RA.
\end{abstract}

Keywords: rheumatoid arthritis, osteoporosis, obesity

\section{INTRODUCTION}

Rheumatoid arthritis (RA) is a chronic inflammatory disease which can lead to permanent disability because of periarticular bone destruction. Modern management principles (1) include early detection and treatment, shared therapeutic decisions, treating to target (aiming for persistent remission) and improving symptoms (e.g. pain) and quality of life. Glucocorticoids are widely used to control RA symptoms, especially at diagnosis, when conventional synthetic disease-modifying anti-rheumatic drugs (csDMARDs) are started, but also whenever the clinical situation calls for their anti-inflammatory effect (e.g. switching DMARDs, disease flares).
For this purpose, rheumatologists most often use low doses of glucocorticoids (less than $7.5 \mathrm{mg}$ /day prednison equivalent) for short periods of time and taper these drugs as soon as the symptoms are controlled by DMARDs. Unfortunately, glucocorticoids are associated with prevalent osteoporosis and fragility fracture risk, since they stimulate bone resorption and inhibit new bone formation (2). This impact of glucocorticoids on bone metabolism is potentially reversible if the exposure ceases and if proper treatment is started early (3). Depending on the clinical case, pharmacological prophylaxis and treatment of glucocorticoid-induced osteoporosis makes use of all available therapeutic principles (optimal calcium 
and vitamin D intake, bisphosphonates, teriparatide, raloxifen, denosumab) (4). Aside from the need of glucocorticoids, RA clusters several other significant factors associated with osteoporosis, such as persistent systemic inflammation of non-responsive or untreated disease (5), prevalent menopause and premature menopause (6), smoking (7), depression $(8,9)$, disability caused by joint damage and chronic pain, fall risk (10) and body composition alterations: on one hand, RA-induced low body mass index (BMI) $(11,12)$ and sarcopenia $(13,14)$, and on the other hand increased prevalence of obesity (15). In fact, two parallel pathogenic processes of bone loss take place during RA course: a local, periarticular bone loss, which is an early radiological sign of RA, and a general (whole body) bone loss (16), leading to osteoporosis and subsequent fragility fractures. The result is that both women and men with RA present a higher prevalence of osteoporosis $(17,18)$, a significantly higher bone loss rate compared to age-matched controls (19), especially in patients with positive rheumatoid factor (RF) and anti-citrullinated protein antibodies (ACPA) (20), higher disease duration (17), low quality of life (17) and exposure to glucocorticoids (21). Since preventing osteoporosis and fragility fractures is one of the main goals in RA management in real world medical environments, we aim to identify clinical RA characteristics which can predict the development of these conditions, in order to guide early therapeutic intervention. For this purpose, the objective of this current initial phase of the study is to retrospectively screen RA phenotype characteristics associated with osteoporosis, which will be prospectively studied for prediction in a subsequent second phase of the study.

\section{METHODS}

\section{Patients}

The study included patients diagnosed with RA by their attending rheumatologists, who also fulfilled the 2010 American College of Rheumatology and European League Against Rheumatism classification criteria (22). All RA patients who came to the rheumatology department in the random order of presentation between January and July 2018 were retrospectively screened for the following exclusion criteria: age under 18 years; overlap syndromes of RA; secondary causes of osteoporosis (cancer, celiac disease, chronic liver disease, chronic pancreatitis, chronic renal disease, chronic obstructive pulmonary disease, Cushing's disease, cystic fibrosis, diabetes mellitus, hemoglobinopathies, heparin treatment, hypogonadism, hyperthyroidism, inflammatory bowel disease, immobility, malabsorption); lack of data regarding osteoporosis status. Written informed consent for medical procedures and scientific use of data is given each time a patient visits the clinic. The
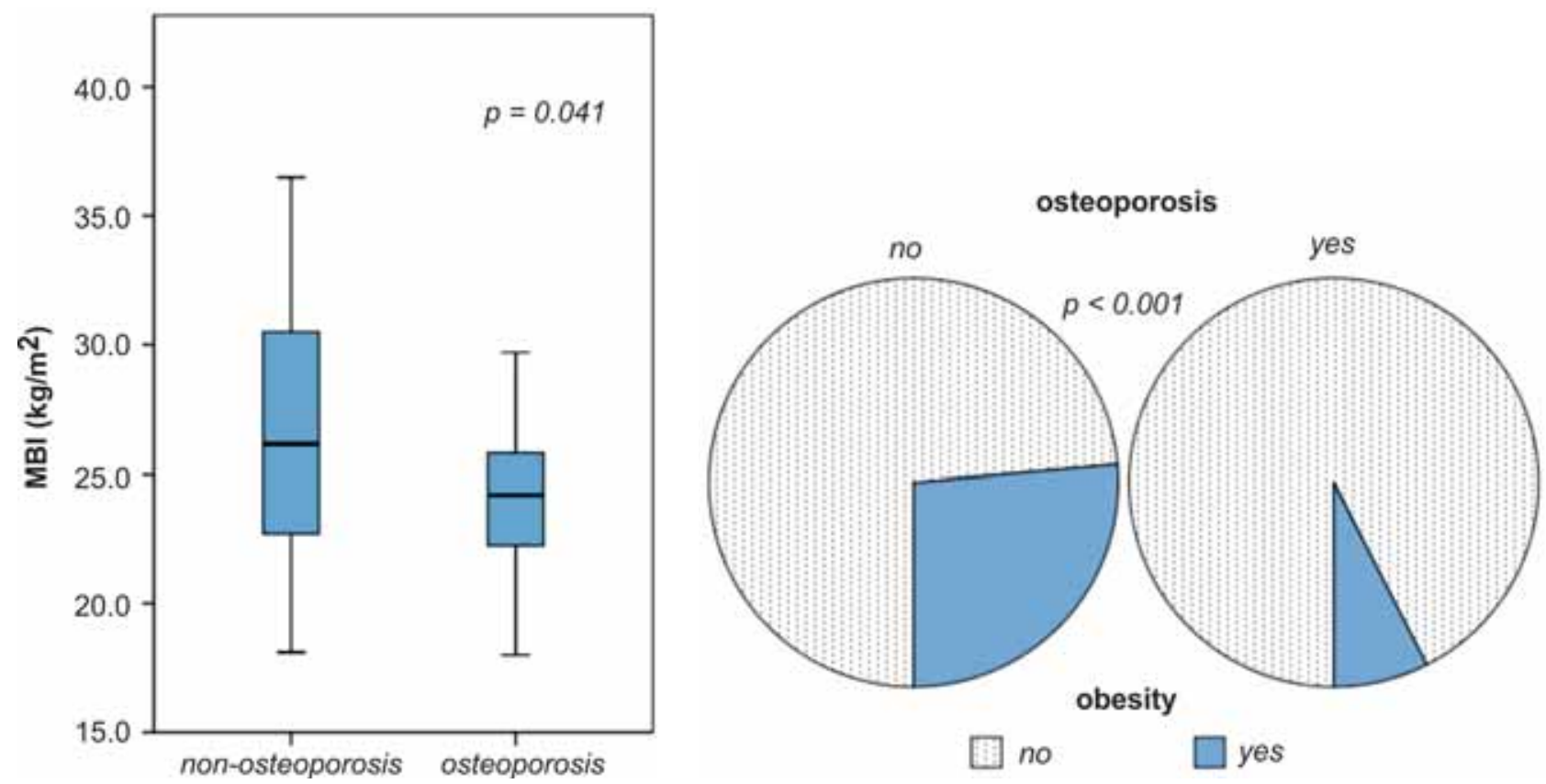

FIGURE 1. Comparison of rheumatoid arthritis patients with and without osteoporosis: the median body mass index difference (left pane: $23.8 \mathrm{~kg} / \mathrm{m}^{2}$ compared to $29.6 \mathrm{~kg} / \mathrm{m}^{2} ; p=0.041$, Mann Whitney test) and the difference of obesity prevalence (right pane: $7.5 \%$ compared to $25.7 \% ; p=0.001, X^{2}$ test; prevalence ratio $=3.43$ ). 
protocol was approved by the local ethics committee.

\section{Variables}

Demographic data (age, gender, dwelling, education level, smoking), anthropometric data (weight, height), RA-specific data (disease duration, disease activity score - DAS28, health assessment questionnaire - HAQ (23), treatment), serology (RF, ACPA), acute phase reactants (erythrocyte sedimentation rate - ESR; C-reactive protein - CRP), hematology tests (hemoglobin, hematocrit), osteoporosis data (diagnosis, lumbar and $\mathrm{T}$ scores, anti-osteoporosis treatment, total serum calcium) and comorbidities (arterial hypertension - AHT; type 2 diabetes mellitus - T2DM; dyslipidemia; carotid artery disease CAD) were collected retrospectively and cross-sectionally from the first (and most frequently the only) observation sheet of each patient within the study timeframe. BMI was either transcribed or calculated by dividing weight to square height. Obesity was defined as a BMI equal to or above $30 \mathrm{~kg} / \mathrm{m}^{2}$ (24). DAS28 was either collected from the observation sheets or calculated using four variables (25): number of tender joints, number of swollen joints, patient global assessment on a visual analogue scale and CRP. Based on DAS28 values, the following disease activity categories were defined: remission (DAS28 < 2.6); low disease activity (LDA; $2.6 \leq$ DAS28 < 3.2); moderate disease activity (MDA; 3.2 $\leq$ DAS2 $<5.1$ ) and high disease activity (HDA; DAS28 $\geq 5.1$ ). Positive serology was defined if RF and ACPA were above the laboratory's upper limit of normal (ULN): $15 \mathrm{U} / \mathrm{mL}$ (turbidimetry method) and respectively $20 \mathrm{U} / \mathrm{mL}$ (enzyme-linked immunosorbent assay method). Inflammation was defined if either ESR (ULN: $30 \mathrm{~mm} / \mathrm{h}$; Westergren method) or CRP (ULN: $5 \mathrm{mg} / \mathrm{L}$; turbidimetry method) were above the laboratory's ULN. Anemia was defined as hemoglobin levels below the gender-specific laboratory lower limit of normal (LLN), respectively $11 \mathrm{~g} /$ $\mathrm{dL}$ for women and $12 \mathrm{~g} / \mathrm{dL}$ for men (flow cytometry with fluorescence method). Hypocalcemia was defined for values bellow the laboratory's LLN, namely $8.8 \mathrm{mg} / \mathrm{dL}$ (spectrophotometry method). Osteoporosis was defined in four non-conflicting instances: the patient had a diagnosis of osteoporosis according to the attending physician, without mention of $\mathrm{T}$ scores and treatment; the patient had active anti-osteoporosis treatment (bisphosphonates, denosumab, teriparatide, calcitonin, estrogens, raloxifen); the patient had a recorded lumbar or hip T score of -2.5 standard deviations (SD) or less (26); the patient had a history of fragility fracture. All the dual energy $\mathrm{X}$-ray absorptiometry scans were performed by a single certified clinical densitometrist $(<0.5 \%$ coefficient of variation) using a Lexxos C05LX223 machine, which was calibrated daily according to the manufacturer's indications. AHT was defined if: the patient had a diagnosis of AHT according to the attending physician; the patient had recorded antihypertensive treatment; the patient has at least two recorded blood pressure measurements of at least 140/90 mmHg (27). T2DM was defined if: the patient had a diagnosis of T2DM according to the attending physician; the patient had recorded antidiabetic treatment; the patient has at least two recorded fasting plasma glucose levels of $126 \mathrm{mg} / \mathrm{dL}$ or more or one recorded value of $200 \mathrm{mg} / \mathrm{dL}$ or more (28). Dyslipidemia was defined if: the patient had a diagnosis of dyslipidemia according to the attending physician; the patient had recorded cholesterol-lowering treatment; the patient had a recorded fasting total cholesterol of $200 \mathrm{mg} / \mathrm{dL}$ or above, serum low-density lipoproteins of $100 \mathrm{mg} / \mathrm{dL}$ or above or serum triglycerides of $150 \mathrm{mg} / \mathrm{dL}$ or more (29). CAD was defied if the patient had a diagnosis of CAD according to the attending physician or if the patient had a recorded imaging study of the extra-cranial internal carotid artery with $50 \%$ stenosis or more (30).

\section{Statistics}

Distribution normality was assessed using descriptive statistics, normality plots and Lillefors corrected Kolmogorov-Smirnov tests. Normally distributed continuous variables were reported as "mean (SD)", while non-normally distributed variables were expressed as "median (interquartile range)". Qualitative variables were expressed as "absolute frequency (percentage of subgroup)". The differences of continuous variables between RA patients with and without osteoporosis were assessed by Mann Whitney tests, while the differences of nominal variables between these subgroups were assessed with $\chi^{2}$ or Fisher's exact tests. The correlations of continuous variables were studied using bivariate Spearman tests and partial correlation controlling for recorded confounders. Prevalence ratio was calculated by dividing the prevalence of obesity among patients with or without osteoporosis. All tests were considered significant if $\mathrm{p}<0.05$ and 
were done using IBM SPSS v.20 (IBM Inc., Armonk, New York, 2010) for Windows.

\section{RESULTS}

\section{General characteristics}

The sample included 149 RA patients, 40 of which had osteoporosis (26.8\%; Table 1). Exactly 84 patients $(56.4 \%)$ were on glucocorticoids for a median of 2.0 (2.4) years with a median dose of 5.0 (5.0) mg prednisone equivalent. All RA patients were receiving csDMARDs: 89 (59.7\%) were taking methotrexate; 32 (21.5\%) had leflunomide; 30 (20.1\%) were receiving hydroxychloroquine and $20(13.4 \%)$ had sulfasalazine. Most of the patients (117, 78.5\%) had csDMARD monotherapy, while 27 patients $(18.1 \%)$ had double therapy and 5 patients $(3.4 \%)$ had triple therapy. Only 11 patients $(7.4 \%)$ were receiving biologic agents: 4 (36.4\%) with rituximab, 3 $(27.3 \%)$ with etanercept, $3(27.3 \%)$ with infliximab and 1 patient $(9.1 \%)$ with adalimumab.

Even though there were only 40 patients with osteoporosis in the whole sample, 83 patients $(55.7 \%)$ were recommended calcium and vitamin D supplements. Of the 40 patients with osteoporosis, only 33 patients (82.5\% of the osteoporosis subgroup) were receiving active pharmacological treatment: 26 patients $(78.8 \%$ of the treated patients) on bisphosphonates, 4 patients $(12.1 \%)$ on denosumab, 2 patients $(6.1 \%)$ on strontium ranelate and 1 patient $(3.0 \%)$ on teriparatide.

\section{Comparison of RA patients with and without osteoporosis}

Compared to RA patients without osteoporosis (patients with osteopenia or normal bone mineral density - BMD), RA patients with osteoporosis were significantly older, with lower BMI and longer disease duration (Table 2).

TABLE 2. Differences of continuous variables between $R A$ patients with and without osteoporosis $(n=149)$

\begin{tabular}{|l|c|c|c|}
\hline & \multicolumn{2}{|c|}{ Osteoporosis } & \\
\hline & no $(\mathbf{n}=109)$ & yes $(\mathbf{n}=40)$ & $\mathbf{p}$ \\
\hline age (years) & $56.0(7.0)$ & $71.0(11.0)$ & 0.001 \\
\hline BMI (kg/m²) & $29.6(6.1)$ & $23.8(4.9)$ & 0.041 \\
\hline RA dura on (years) & $11.0(17.0)$ & $17.0(7.5)$ & 0.035 \\
\hline ESR (mm/h) & $34.0(39.0)$ & $42.0(42.0)$ & 0.852 \\
\hline CRP $(\mathrm{mg} / \mathrm{L})$ & $4.7(6.4)$ & $6.1(19.2)$ & 0.636 \\
\hline RF $(\mathrm{U} / \mathrm{mL})$ & $129.6(397.3)$ & $56.8(87.5)$ & 0.647 \\
\hline ACPA (U/mL) & $104.1(141.5)$ & $69.2(93.6)$ & 0.566 \\
\hline hemoglobin (g/dL) & $14.2(1.0)$ & $12.7(2.1)$ & 0.111 \\
\hline hematocrit $(\%)$ & $42.8(3.7)$ & $39.5(4.2)$ & 0.163 \\
\hline HAQ & $1.5(1.4)$ & $1.0(0.8)$ & 0.533 \\
\hline DAS28 & $4.1(2.1)$ & $3.9(1.7)$ & 0.687 \\
\hline TSCa ${ }^{2+}(\mathrm{mg} / \mathrm{dL})$ & $9.4(0.9)$ & $9.1(0.7)$ & 0.846 \\
\hline
\end{tabular}

Notes: non-normally distributed data are reported as "median (interquartile range)"; $p$ values represent the significance level of Mann Whitney tests; see Methods section of specific definitions of reported items; abbreviations: ACPA - anti-citrullinated protein antibodies; BMI - body mass index; CRP - C-reactive protein; DAS - disease activity score; ESR - erythrocyte sedimentation rate; $\mathrm{HAQ}$ - health assessment questionnaire; RA - rheumatoid arthritis; RF - rheumatoid factor; $\mathrm{TSCa}^{2+}$ - total serum calcium.

Similarly, compared to RA patients without osteoporosis, RA patients with osteoporosis had a higher

TABLE 1. General characteristics of the sample $(n=149)$

\begin{tabular}{|c|c|c|c|}
\hline age (years) & $60.8 \pm 12.9$ & RA duration (years) & $7.0(11.0)$ \\
\hline women $(n, \%)$ & $121(81.2 \%)$ & $\mathrm{HAQ}$ & $1.4 \pm 0.7$ \\
\hline urban dwelling $(n, \%)$ & $90(60.4 \%)$ & DAS28 & $4.1 \pm 1.2$ \\
\hline college $(n, \%)$ & $21(14.1 \%)$ & remission $(n, \%)$ & $12(8.1 \%)$ \\
\hline smoking (n, \%) & 27 (18.1\%) & $\operatorname{LDA}(n, \%)$ & $6(4.1 \%)$ \\
\hline $\mathrm{BMI}\left(\mathrm{kg} / \mathrm{m}^{2}\right)$ & $25.9 \pm 4.8$ & $\operatorname{MDA}(n, \%)$ & $47(31.5 \%)$ \\
\hline obesity (n, \%) & $31(20.8 \%)$ & $\operatorname{HDA}(n, \%)$ & $14(9.4 \%)$ \\
\hline $\mathrm{TSCa}^{2+}(\mathrm{mg} / \mathrm{dL})$ & $9.2 \pm 0.4$ & $\mathrm{RF}(\mathrm{U} / \mathrm{mL})$ & $58.8(146.3)$ \\
\hline hypocalcemia (n, \%) & $7(4.7 \%)$ & posi ve RF $(n, \%)$ & 107 (71.8\%) \\
\hline lumbar BMD $\left(\mathrm{g} / \mathrm{m}^{2}\right)$ & $0.81 \pm 0.14$ & ACPA $(\mathrm{U} / \mathrm{mL})$ & 101.9 (152.1) \\
\hline lumbar T score (SD) & $-1.8 \pm 0.9$ & posi ve ACPA $(n, \%)$ & $101(67.8 \%)$ \\
\hline hip T score (SD) & $-1.8 \pm 1.0$ & $\operatorname{ESR}(\mathrm{mm} / \mathrm{h})$ & $40.0(48.0)$ \\
\hline osteoporosis (n, \%) & $40(26.8 \%)$ & $\mathrm{CRP}(\mathrm{mg} / \mathrm{L})$ & $11.0(23.3)$ \\
\hline AHT $(n, \%)$ & $79(53.1 \%)$ & inflamma on $(n, \%)$ & $120(80.5 \%)$ \\
\hline T2DM (n, \%) & $8(5.4 \%)$ & hemoglobin (g/dL) & $12.8 \pm 1.5$ \\
\hline dyslipidemia (n, \%) & $62(41.6 \%)$ & hematocrit (\%) & $38.8 \pm 4.2$ \\
\hline $\operatorname{CAD}(n, \%)$ & $19(12.8 \%)$ & anemia $(n, \%)$ & $18(12.1 \%)$ \\
\hline
\end{tabular}

Notes: normally-distributed data are reported as "mean \pm SD"; non-normally distributed data are reported as "median (interquartile range)"; see Methods section of specific definitions of reported items; abbreviations: ACPA - anti-citrullinated protein antibodies; AHT - arterial hypertension; BMD - bone mineral density; BMI - body mass index; CAD - carotid artery disease; CRP - C-reactive protein; DAS - disease activity score; ESR - erythrocyte sedimentation rate; $\mathrm{HAQ}$ - health assessment questionnaire; HDA - high disease activity; LDA - low disease activity; MDA - moderate disease activity; RA - rheumatoid arthritis; RF - rheumatoid factor; SD - standard deviation; T2DM - type 2 diabetes mellitus; TSCa ${ }^{2+}$ - total serum calcium. 
prevalence of rural dwelling, smoking, inflammation, ACPA positivity, glucocorticoid treatment and CAD, but a lower prevalence of obesity (Table 3).

TABLE 3. Differences of nominal variables between $R A$ patients with and without osteoporosis $(n=149)$

\begin{tabular}{|l|c|c|c|}
\hline \multirow{2}{*}{} & \multicolumn{2}{|c|}{ Osteoporosis } & \\
\cline { 2 - 4 } & no (n=109) & yes (n= 40) & $p$ \\
\hline men (n, \%) & $23(21.1 \%)$ & $5(12.5 \%)$ & 0.336 \\
\hline rural dwelling (n, \%) & $31(28.4 \%)$ & $28(70.0 \%)$ & 0.012 \\
\hline college (n, \%) & $13(11.9 \%)$ & $8(20.0 \%)$ & 0.691 \\
\hline smoking (n, \%) & $11(10.1 \%)$ & $16(37.5 \%)$ & 0.030 \\
\hline obesity (n, \%) & $28(25.7 \%)$ & $3(7.5 \%)$ & 0.001 \\
\hline inflammation (n, \%) & $81(74.3 \%)$ & $40(100.0 \%)$ & 0.039 \\
\hline RF positive (n, \%) & $76(69.7 \%)$ & $31(77.5 \%)$ & 0.372 \\
\hline ACPA positive (n, \%) & $65(59.6 \%)$ & $36(90.0 \%)$ & 0.033 \\
\hline glucocorticoids (n, \%) & $50(45.9 \%)$ & $34(85.0 \%)$ & 0.044 \\
\hline methotrexate (n, \%) & $66(60.6 \%)$ & $23(57.5 \%)$ & 0.400 \\
\hline leflunomide (n, \%) & $22(20.2 \%)$ & $9(22.5 \%)$ & 0.929 \\
\hline sulfasalazine (n, \%) & $14(12.8 \%)$ & $6(15.0 \%)$ & 0.600 \\
\hline $\begin{array}{l}\text { hydroxychloroquine } \\
\text { (n, \%) }\end{array}$ & $22(20.2 \%)$ & $8(20.0 \%)$ & 0.970 \\
\hline $\begin{array}{l}\text { csDMARD monotherapy } \\
\text { (n, \%) }\end{array}$ & $84(77.1 \%)$ & $33(82.5 \%)$ & 0.543 \\
\hline $\begin{array}{l}\text { csDMARD dual therapy } \\
\text { (n, \%) }\end{array}$ & $22(20.2 \%)$ & $5(12.5 \%)$ & 0.408 \\
\hline $\begin{array}{l}\text { csDMARD triple therapy } \\
\text { (n, \%) }\end{array}$ & $3(2.8 \%)$ & $2(5.0 \%)$ & 0.579 \\
\hline biologic DMARDs (n, \%) & $8(7.3 \%)$ & $3(7.5 \%)$ & 0.923 \\
\hline anemia (n, \%) & $12(11.0 \%)$ & $6(15.0 \%)$ & 0.679 \\
\hline HDA (n, \%) & $8(7.3 \%)$ & $6(15.0 \%)$ & 0.135 \\
\hline hypocalcemia (n, \%) & $5(4.6 \%)$ & $2(5.0 \%)$ & 0.655 \\
\hline AHT (n, \%) & $55(50.4 \%)$ & $24(60.0 \%)$ & 0.478 \\
\hline T2DM (n, \%) & $6(5.5 \%)$ & $2(5.0 \%)$ & 0.272 \\
\hline dyslipidemia (n, \%) & $42(38.5 \%)$ & $20(50.0 \%)$ & 0.242 \\
\hline CAD (n, \%) & $9(8.3 \%)$ & $10(25.0 \%)$ & 0.038 \\
\hline Notes: nomina vaia) & & & \\
\hline
\end{tabular}

Notes: nominal variables are reported as "absolute frequency (percent of subgroup)"; $p$ values represent the significance level of $\chi^{2}$ tests; see Methods section of specific definitions of reported items; abbreviations: ACPA - anti-citrullinated protein antibodies; AHT - arterial hypertension; CAD - carotid artery disease; csDMARDs - conventional synthetic disease-modifying anti-rheumatic drugs; HDA - high disease activity; RA - rheumatoid arthritis; RF - rheumatoid factor; T2DM type 2 diabetes mellitus.

Compared with RA patients with normal acute phase reactants, patients with inflammation had a significantly lower median lumbar BMD (0.73 g/ $\mathrm{cm}^{2}$ compared to $\left.0.83 \mathrm{~g} / \mathrm{cm}^{2} ; \mathrm{p}=0.046\right)$. Lumbar BMD was significantly and positively correlated with BMI $(r=0.294, p=0.045)$ and with the titer of $R F(r=0.311, p=0.033)$, correlations with remained significant after controlling for age, gender and disease duration. Lumbar $\mathrm{T}$ scores were significantly correlated with age $(r=-0.272 ; p=0.010)$ and hematocrit $(r=0.240 ; p=0.025)$, while hip T scores were significantly correlated with age $(r=-0.337$; $\mathrm{p}=0.001)$, hematocrit $(\mathrm{r}=0.216 ; \mathrm{p}=0.044)$ and hemoglobin $(r=0.298 ; p=0.005)$. However, partial correlations of these variables, controlling for age, gender and disease duration, rendered insignificant correlations $(p>0.08)$.

\section{DISCUSSION}

Three main associations of osteoporosis we observed among RA patients: osteoporosis showed a negative association with obesity and positive associations with glucocorticoids, inflammation, ACPA positivity and cardiovascular disease (as represented by CAD).

More specifically, our RA patients with osteoporosis had significantly lower BMI, while RA patients without osteoporosis had more than 3 times the prevalence of obesity among RA patients with osteoporosis. It is known that low BMI represents an independent and significant risk factor for osteoporosis $(31,32)$, therefore one can suppose a protective effect of body fat on BMD. This hypothesis was proven: for example, Mazocco et al. (33) reported, as we observed, that the prevalence of osteoporosis among obese subjects is significantly lower than that of normal-weight and overweight subjects, while Barrera et al. (34), studying femoral neck BMD, reported that obesity incurs a three-fold reduction in the risk for osteoporosis compared to persons with normal weight. Further elaborating the link between obesity and osteoporosis and thus strengthening, in the limits of epidemiological observation, the probability of a protective effect of obesity on BMD, Oldroyd et al. (35) reported that it is limited by an upper threshold of $40 \mathrm{~kg} / \mathrm{m}^{2}$, above which the prevalence of osteoporosis increases even among obese subjects, proposing a mechanical explanation of failing weight bearing in the morbidly obese to explain fragility fractures among these patients. Another clue to the deterministic relationship of obesity and osteoporosis is the report of Compston et al. (11), which observed that the influence of obesity on BMD varies according to site: obesity protects against vertebral, hip and radius fragility fractures, but it is associated with an increased risk of ankle fragility fractures. All these results suggest a causal relationship between osteoporosis risk reduction in obesity (36): the conditions are significantly and negatively correlated; the effect is consistently reported in different geographical areas and in genetically different human populations; the relationship is specific, in 
the sense that there are no other diseases known to reduce the risk of osteoporosis; the different risk of osteoporosis along the BMI scale clearly indicates a biological gradient and is coherent with most of the available knowledge; biological plausibility can be invoked by citing estrogen synthesis by adipose tissue (37). However, there is insufficient data regarding experimentation (for example, the observation of increasing risk of osteoporosis in formerly obese persons who lost weight) and temporality (whether obesity increases the risk for osteoporosis of formerly normal-weight persons who became obese). Notable doubts about the causal protective effect of obesity on osteoporosis have been raised during the last decade. For example, completely opposite of what we discussed so far, Greco et al. (38) reported that obesity was positively associated with osteoporosis in their sample of subjects, while Neglia et al. (39) reported a 1.46 adjusted odds ratio for osteoporosis in their obese subjects. Recent genetic research lead to the identification of genes and genetic polymorphisms which are associated both with osteoporosis and obesity $(40,41)$. Therefore, it seems that the relationship between the two conditions has two ways and hints at the hypothesis that not all fat is protective against osteoporosis. Indeed, there are reports which prove that abdominal obesity is inversely correlated with BMD and it increases the risk of fragility fractures (42-44). These observations are plausible if the exocrine capacity of excess adipose tissue is taken into consideration: high levels of adipokines, such as leptin (45), and of pro-inflammatory cytokines, such as tumor necrosis factor $\alpha$ (46), are known to decrease BMD and to be associated with abdominal obesity. The same abdominal obesity is linked not only to osteoporosis, but more thoroughly to negative cardiovascular outcomes (47), as we observed in our sample the higher prevalence of CAD among osteoporosis patients, and it may represent the confounding element of our previously observed correlation of bone density and cardiovascular risk (48). In order to elucidate the link between osteoporosis and obesity, which cannot be ultimately done epidemiologically, fundamental research should concentrate on the identification and description of adipose tissue exocrine products and their effects on bone tissue and endothelia. RA may not be the optimal medium to investigate this connection, since it is a condition which by itself or through its treatment influences adipose tissue, cardiovascular risk and bone metabolism in the same time, making it hard to differentiate the individual deterministic relationships between these states. Therefore, the second phase of this study is planned to prospectively observe the influence of trunk adiposity and BMI variation on BMD variation and osteoporosis development, both in RA patients and healthy controls.

Another discussion point is the positive association of osteoporosis with ACPA positivity in our study: ACPA positivity was 1.5 times more frequent among patients with osteoporosis compared to RA patients without osteoporosis (Table 3). The literature confirms our findings: systematically, ACPA positive RA patients have lower BMD of regular regions of interest (spine, hip, forearm) compared to ACPA negative RA patients $(49,50)$, translating in a higher fracture risk for these ACPA positive patients independently of ACPA titers (51). However, the effect of ACPA on osteoporosis in RA seems to be dependent on ACPA titers $(52,53)$, following a linear relationship (54), which determined some authors to hypothesize that RA-specific autoimmunity causally determines systemic bone loss $(49,50)$ and that lowering ACPA titers would improve bone mass (52). We consider that the evidence supporting such a direct causal relationship is not compelling. Rather, a confounding RA-specific process determines both ACPA positivity and systemic bone loss, as Wysham et al. recently suggested (54). Fundamental research seems to support this view, proving that ACPA positivity and osteoporosis in RA have common biochemical (such as interleukin 6 (55) and receptor activator of nuclear factor $\kappa-B$ ligand (56)) or even genetic determinants (such as vitamin D receptor polymorphisms (57)). A more plausible explanation for this epidemiologic association of osteoporosis and ACPA in RA should be sought in the field of osteoimmunology: osteoclasts respond to the same pro-inflammatory signals which drive disease activity in RA.

The relevance of our results could be influence by a series of study limitations: the retrospective and uncontrolled design; the relatively low sample number which may be further biased for selection, since all of the patients were included in the study upon their request of medical services; the lack of information regarding significant confounding variables (such as physical activity and diet); to inability to establish whether the significantly lower BMI of our RA patients with osteoporosis is random or partially caused by RA itself. 


\section{CONCLUSION}

Osteoporosis among RA patients seems to be associated with a lower prevalence of BMI-defined obesity, but with a higher prevalence of disease se-

\section{REFERENCES}

1. Smolen J.S., Landewe R., Bijlsma J., Burmester G., Chatzidionysiou K., Dougados M. et al. EULAR recommendations for the management of rheumatoid arthritis with synthetic and biological disease-modifying antirheumatic drugs: 2016 update. Ann Rheum Dis. 2017; 76(6):960-77.

2. Canalis E., Mazziotti G., Giustina A., Bilezikian J.P. Glucocorticoid-induced osteoporosis: pathophysiology and therapy. Osteoporos Int. 2007; 18(10):1319-28.

3. Overman R.A., Gourlay M.L., Deal C.L., Farley J.F., Brookhart M.A., Layton J.B. Fracture rate associated with quality metricbased anti-osteoporosis treatment in glucocorticoid-induced osteoporosis. Osteoporos Int. 2015; 26(5):1515-24.

4. Buckley L., Guyatt G., Fink H.A., Cannon M., Grossman J., Hansen K.E. et al. 2017 American College of Rheumatology Guideline for the Prevention and Treatment of GlucocorticoidInduced Osteoporosis. Arthritis Rheumatol. 2017;69(8):1521-37.

5. Roux C. Osteoporosis in inflammatory joint diseases. Osteoporos Int. 2011; 22(2):421-33.

6. Banas T., Hajdyla-Banas I., Pitynski K., Nieweglowska D., Juszczyk G., Ludwin A. et al. Age at natural menopause in women on long-term methotrexate therapy for rheumatoid arthritis. Menopause. 2016; 23(10):1130-8.

7. Chang K., Yang S.M., Kim S.H., Han K.H., Park S.J., Shin J.I. Smoking and rheumatoid arthritis. Int J Mol Sci. 2014; 15(12):22279-95.

8. Kawada T. The prevalence of depression in rheumatoid arthritis: a systematic review and meta-analysis. Rheumatology (Oxford). 2014; 53(3):578.

9. Matcham F., Rayner L., Steer S., Hotopf M. The prevalence of depression in rheumatoid arthritis: a systematic review and metaanalysis: reply. Rheumatology (Oxford). 2014; 53(3):578-9.

10. Brenton-Rule A., Dalbeth N., Bassett S., Menz H.B., Rome K. The incidence and risk factors for falls in adults with rheumatoid arthritis: a systematic review. Semin Arthritis Rheum. 2015; 44(4):389-98.

11. Compston J.E., Flahive J., Hosmer D.W., Watts N.B., Siris E.S., Silverman S. et al. Relationship of weight, height, and body mass index with fracture risk at different sites in postmenopausal women: the Global Longitudinal study of Osteoporosis in Women (GLOW). J Bone Miner Res. 2014;29(2):487-93.

12. Albrecht K., Richter A., Callhoff J., Huscher D., Schett G., Strangfeld $\mathbf{A}$. et al. Body mass index distribution in rheumatoid arthritis: a collaborative analysis from three large German rheumatoid arthritis databases. Arthritis Res Ther. 2016;18:149.

13. Ngeuleu A., Allali F., Medrare L., Madhi A., Rkain H., HajjajHassouni N. Sarcopenia in rheumatoid arthritis: prevalence, influence of disease activity and associated factors. Rheumatol Int. 2017;37(6):1015-20

14. Di Monaco M., Vallero F., Di Monaco R., Tappero R. Prevalence of sarcopenia and its association with osteoporosis in 313 older women following a hip fracture. Arch Gerontol Geriatr. 2011; 52(1):71-4.

15. Stavropoulos-Kalinoglou A., Metsios G.S., Koutedakis Y., Kitas G.D. Obesity in rheumatoid arthritis. Rheumatology (Oxford). 2011; 50(3):450-62.

16. Popescu C., Bojinca V., Opris D., Ionescu R. Dual X-Ray Absorptiometry Whole Body Composition of Bone Tissue in Rheumatoid Arthritis - a Cross-Sectional Study. Maedica (Buchar). 2015;10(1):19-26

17. Lee J.H., Sung Y.K., Choi C.B., Cho S.K., Bang S.Y., Choe J.Y. et al. The frequency of and risk factors for osteoporosis in Korean verity markers (treatment with glucocorticoids, inflammation and ACPA positivity). Gain of adipose tissue and loss of bone tissue seem to be antagonistic and parallel body composition alterations in RA.

Conflict of interest: none declared Financial support: none declared

patients with rheumatoid arthritis. BMC Musculoskelet Disord. 2016;17:98.

18. Piao H.H., Zhang K.Q., Tang Z.H. Association between rheumatoid arthritics and osteoporosis among Chinese men, a community based study. Int J Clin Exp Med. 2015; 8(9):16592-8.

19. Hauser B., Riches P.L., Wilson J.F., Horne A.E., Ralston S.H. Prevalence and clinical prediction of osteoporosis in a contemporary cohort of patients with rheumatoid arthritis. Rheumatology (Oxford). 2014;53(10):1759-66.

20. Mobini M., Kashi Z., Ghobadifar A. Prevalence and associated factors of osteoporosis in female patients with rheumatoid arthritis. Caspian J Intern Med. 2012; 3(3):447-50.

21. Coulson K.A., Reed G., Gilliam B.E., Kremer J.M., Pepmueller P.H. Factors influencing fracture risk, T score, and management of osteoporosis in patients with rheumatoid arthritis in the Consortium of Rheumatology Researchers of North America (CORRONA) registry. J Clin Rheumatol. 2009;15(4):155-60.

22. Aletaha D., Neogi T., Silman A.J., Funovits J., Felson D.T., Bingham C.O., 3rd, et al. 2010 rheumatoid arthritis classification criteria: an American College of Rheumatology/European League Against Rheumatism collaborative initiative. Ann Rheum Dis. 2010; 69(9):1580-8.

23. Fries J.F., Spitz P., Kraines R.G., Holman H.R. Measurement of patient outcome in arthritis. Arthritis Rheum. 1980; 23(2):137-45.

24. Physical status: the use and interpretation of anthropometry. Report of a WHO Expert Committee. World Health Organ Tech Rep Ser. 1995; 854:1-452.

25. Prevoo M.L., van, t Hof M.A., Kuper H.H., van Leeuwen M.A., van de Putte L.B., van Riel P.L. Modified disease activity scores that include twenty-eight-joint counts. Development and validation in a prospective longitudinal study of patients with rheumatoid arthritis. Arthritis Rheum. 1995; 38(1):44-8.

26. NIH Consensus Development Panel on Osteoporosis Prevention, Diagnosis, and Therapy, March 7-29, 2000: highlights of the conference. South Med J. 2001; 94(6):569-73.

27. Mancia G., Fagard R., Narkiewicz K., Redon J., Zanchetti A., Bohm M. et al. 2013 ESH/ESC Practice Guidelines for the Management of Arterial Hypertension. Blood Press. 2014; 23(1):3-16.

28. Alberti K.G., Zimmet P.Z. Definition, diagnosis and classification of diabetes mellitus and its complications. Part 1: diagnosis and classification of diabetes mellitus provisional report of a WHO consultation. Diabet Med. 1998;15(7):539-53.

29. Jellinger P.S., Handelsman Y., Rosenblit P.D., Bloomgarden Z.T., Fonseca V.A., Garber A.J. et al. American Association of Clinical Endocrinologists and American College of Endocrinology Guidelines for Management of Dyslipidemia and Prevention of Cardiovascular Disease. Endocr Pract. 2017; 23(Suppl 2):1-87.

30. Aboyans V., Ricco J.B., Bartelink M.E.L., Bjorck M., Brodmann M., Cohnert T. et al. 2017 ESC Guidelines on the Diagnosis and Treatment of Peripheral Arterial Diseases, in collaboration with the European Society for Vascular Surgery (ESVS): Document covering atherosclerotic disease of extracranial carotid and vertebral, mesenteric, renal, upper and lower extremity arteriesEndorsed by: the European Stroke Organization (ESO)The Task Force for the Diagnosis and Treatment of Peripheral Arterial Diseases of the European Society of Cardiology (ESC) and of the European Society for Vascular Surgery (ESVS). Eur Heart J. 2018;39(9):763-816

31. Asomaning K., Bertone-Johnson E.R., Nasca P.C., Hooven F., Pekow P.S. The association between body mass index and 
osteoporosis in patients referred for a bone mineral density examination. J Womens Health (Larchmt). 2006;15(9):1028-34.

32. Cui R., Zhou L., Li Z., Li Q., Qi Z., Zhang J. Assessment risk of osteoporosis in Chinese people: relationship among body mass index, serum lipid profiles, blood glucose, and bone mineral density. Clin Interv Aging. 2016;11:887-95.

33. Mazocco L., Chagas P. Association between body mass index and osteoporosis in women from northwestern Rio Grande do Sul. Rev Bras Reumatol Engl Ed. 2017; 57(4):299-305.

34. Barrera G., Bunout D., Gattas V., de la Maza M.P., Leiva L., Hirsch S. A high body mass index protects against femoral neck osteoporosis in healthy elderly subjects. Nutrition. 2004; 20(9):769-71

35. Oldroyd A., Mitchell K., Bukhari M. The prevalence of osteoporosis in an older population with very high body mass index: evidence for an association. Int J Clin Pract. 2014; 68(6):771-4.

36. Hill A.B. The Environment and Disease: Association or Causation? Proc R Soc Med. 1965; 58:295-300.

37. Greco E.A., Lenzi A., Migliaccio S. The obesity of bone. Ther Adv Endocrinol Metab. 2015;6(6):273-86.

38. Greco E.A., Fornari R., Rossi F., Santiemma V., Prossomariti G., Annoscia C. et al. Is obesity protective for osteoporosis? Evaluation of bone mineral density in individuals with high body mass index. Int J Clin Pract. 2010; 64(6):817-20.

39. Neglia C., Argentiero A., Chitano G., Agnello N., Ciccarese R., Vigilanza A. et al. Diabetes and Obesity as Independent Risk Factors for Osteoporosis: Updated Results from the ROIS/ EMEROS Registry in a Population of Five Thousand PostMenopausal Women Living in a Region Characterized by Heavy Environmental Pressure. Int J Environ Res Public Health. 2016; 13(11).

40. Correa-Rodriguez M., Schmidt Rio-Valle J., Rueda-Medina B. SOX6 rs7117858 polymorphism is associated with osteoporosis and obesity-related phenotypes. Eur J Clin Invest. 2018:e13011.

41. Hu Y., Tan L.J., Chen X.D., Liu Z., Min S.S., Zeng Q. et al. Identification of Novel Potentially Pleiotropic Variants Associated With Osteoporosis and Obesity Using the cFDR Method. J Clin Endocrinol Metab. 2018;103(1):125-38.

42. Greco E.A., Francomano D., Fornari R., Marocco C., Lubrano C., Papa V. et al. Negative association between trunk fat, insulin resistance and skeleton in obese women. World J Diabetes. 2013; 4(2):31-9.

43. Sogaard A.J., Holvik K., Omsland T.K., Tell G.S., Dahl C., Schei B. et al. Abdominal obesity increases the risk of hip fracture. A population-based study of 43,000 women and men aged $60-79$ years followed for 8 years. Cohort of Norway. J Intern Med. 2015; 277(3):306-17.

44. Yang S., Nguyen N.D., Center J.R., Eisman J.A., Nguyen T.V. Association between abdominal obesity and fracture risk: a prospective study. J Clin Endocrinol Metab. 2013; 98(6):2478-83.

45. Kontogianni M.D., Dafni U.G., Routsias J.G., Skopouli F.N. Blood leptin and adiponectin as possible mediators of the relation between fat mass and BMD in perimenopausal women. $J$ Bone Miner Res. 2004;19(4):546-51.
46. Zha L., He L., Liang Y., Qin H., Yu B., Chang L. et al. TNF-alpha contributes to postmenopausal osteoporosis by synergistically promoting RANKL-induced osteoclast formation. Biomed Pharmacother. 2018; 102:369-74.

47. Lee C.M., Huxley R.R., Wildman R.P., Woodward M. Indices of abdominal obesity are better discriminators of cardiovascular risk factors than BMI: a meta-analysis. J Clin Epidemiol. 2008; 61(7):646-53.

48. Popescu C., Bojinca V., Opris D., lonescu R. Whole body bone tissue and cardiovascular risk in rheumatoid arthritis. J Osteoporos. 2014; 2014:465987.

49. Bugatti S., Bogliolo L., Vitolo B., Manzo A., Montecucco C., Caporali R. Anti-citrullinated protein antibodies and high levels of rheumatoid factor are associated with systemic bone loss in patients with early untreated rheumatoid arthritis. Arthritis Res Ther. 2016;18(1):226.

50. Guler H., Turhanoglu A.D., Ozer B., Ozer C., Balci A. The relationship between anti-cyclic citrullinated peptide and bone mineral density and radiographic damage in patients with rheumatoid arthritis. Scand J Rheumatol. 2008; 37(5):337-42.

51. Cheng T.T., Yu S.F., Su F.M., Chen Y.C., Su B.Y., Chiu W.C. et al. Anti-CCP-positive patients with RA have a higher 10-year probability of fracture evaluated by $F R A X(R)$ : a registry study of RA with osteoporosis/fracture. Arthritis Res Ther. 2018;20(1):16.

52. Orsolini G., Caimmi C., Viapiana O., Idolazzi L., Fracassi E., Gatti D. et al. Titer-Dependent Effect of Anti-Citrullinated Protein Antibodies On Systemic Bone Mass in Rheumatoid Arthritis Patients. Calcif Tissue Int. 2017;101(1):17-23.

53. Ahmad H.A., Alemao E., Guo Z., lannaccone C.K., Frits M.L., Weinblatt M. et al. Association of Low Bone Mineral Density with Anti-Citrullinated Protein Antibody Positivity and Disease Activity in Established Rheumatoid Arthritis: Findings from a US Observational Cohort. Adv Ther. 2018;35(2):232-42.

54. Wysham K.D., Shoback D.M., Imboden J.B., Jr., Katz P.P. Association of High Anti-Cyclic Citrullinated Peptide Seropositivity and Lean Mass Index With Low Bone Mineral Density in Rheumatoid Arthritis. Arthritis Care Res (Hoboken). 2018;70(7):961-9.

55. Abdel Meguid M.H., Hamad Y.H., Swilam R.S., Barakat M.S. Relation of interleukin-6 in rheumatoid arthritis patients to systemic bone loss and structural bone damage. Rheumatol Int. 2013; 33(3):697-703.

56. Johansson L., Arlestig L., Kokkonen H., Brink M., RantapaaDahlqvist S. An increased concentration of receptor activator of nuclear factor kappa-B ligand pre-dates the onset of rheumatoid arthritis. Rheumatology (Oxford). 2017;56(12):2190-6.

57. Mosaad Y.M., Hammad E.M., Fawzy Z., Abdal Aal I.A., Youssef H.M., EISaid T.O. et al. Vitamin D receptor gene polymorphism as possible risk factor in rheumatoid arthritis and rheumatoid related osteoporosis. Hum Immunol. 2014;75(5):452-61. 